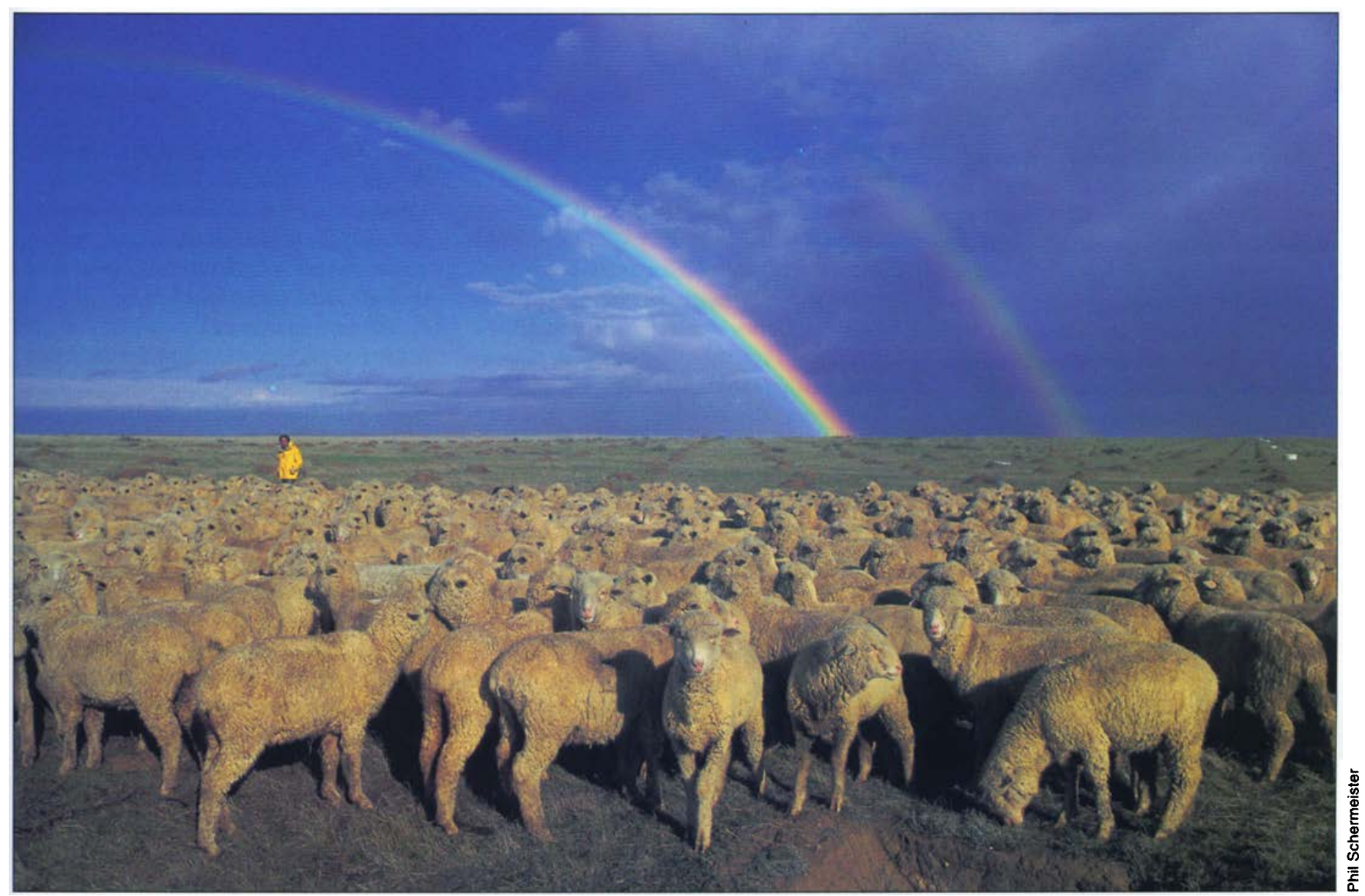

Transgenic sheep, goats, pigs and cattle are now routinely made, although efficiency is low and costs are high. Conventionally bred sheep are herded near Cholame.

\title{
Genetic engineering and cloning may improve milk, livestock production
}

\author{
James D. Murray a Gary B. Anderson
}

In the past, procedures such as artificial insemination and embryo transfer have been used in the genetic manipulation of livestock. Advances in gene and quantitativetrait mapping will enhance these traditional animal-breeding approaches to improve farm animals. By genetically engineering livestock, scientists hope to produce animals with altered traits such as disease resistance, wool growth, body growth and milk composition. Laboratories worldwide have produced transgenic pigs, sheep, goats and cattle, but currently the efficiency of producing the animals remains low and the procedure is expensive.
Within the next few decades, however, genetically engineered dairy cows could become available. Cloning may also be used to duplicate animals with traits that are difficult to capture through traditional breeding practices. By 2025, cloning and breeding of elite animals could be carried out by companies comparable to those that now comprise the artificial insemination industry, which selects and breeds top dairy stock. The acceptance of genetically engineered animals by industry will depend on its economic benefits and whether consumers are prepared to buy the resulting products.
Traditionally, genetic improve1 ment of livestock has been achieved by applying the principles of quantitative genetics and animal breeding. Milk production in dairy cattle, for example, has increased 100 pounds of milk per cow per year due to genetic selection, augmented by artificial insemination. Advances in gene and quantitativetrait mapping in the last decade will ensure that traditional animalbreeding approaches, coupled with newer techniques for markerassisted selection, will be effective in providing continued genetic improvement of livestock. The advent of modern biotechnology also prqvides new avenues for genetic improvement in production animals. 
In the broadest sense, biotechnology includes procedures commonly used in animal production, such as artificial insemination and embryo transfer - both of which have successfully increased the rate of genetic improvement in species. During the past 10 to 15 years, however, the term biotechnology has come to be associated more with molecular-based technologies, such as gene cloning and genetic engineering.

The initial development of techniques for cloning and manipulating gene sequences in the 1970s was followed 10 years later by techniques for producing genetically engineered or transgenic animals, resulting in a paradigm shift in modern biological research (Gordon et al. 1980). Initial successes with transgenic mice were subsequently extended to agricultural species. Laboratories worldwide have produced transgenic pigs, sheep, goats and cattle (Pinkert and Murray 1999). However, the efficiency of producing transgenic ruminants and pigs remains low, the costs are high and the time required to produce and characterize transgenic livestock is long (Wall et al. 1992). For example, determination that the introduced gene is

\section{Glossary}

Backcrossing: crossing an offspring to one or the other of two parental strains or breeds.

DNA: deoxyribonucleic acid, the molecule containing the genetic code.

Embryonic stem (ES) cells: undifferentiated cells derived from embryos and capable of surviving prolonged culture while still contributing to development of viable offspring. Embryonic stem cells can integrate foreign DNA. Laboratory techniques can isolate ES cells that have integrated the transgene at a specific site in the genome.

Expression: production of a protein or other molecule from a gene coded in the DNA.

Gene construct: man-made DNA molecule used to produce a transgenic animal.

Gene knockout: disruption of the function of a specific gene. Currently gene knockouts require ES cell technology.

Gene replacement: substitution of one gene for another. Like knockouts, targeting a particular gene now requires ES cell technology.

Genome: the complete set of genetic information for an individual.

Heritability: measure of the extent to which an observed trait is controlled by genetics as compared to the environment in which the animal lives.

Inbreeding: mating of close relatives, leading to a reduction in genetic di- versity in the offspring and increasing the likelihood of expression of deleterious genes.

Introgression: movement of a gene from one population to another.

In vitro: in the laboratory, outside the organism.

Lysozyme: naturally occurring protein with antimicrobial properties.

Marker-assisted selection: use of DNAbased markers to aid in selective breeding for or against a specific trait.

Micelle: assembly of milk proteins into a particle.

Overexpression: increased expression of a gene above the normally observed level.

Phenotype: physical characteristics of an individual.

Promoter: part of a gene that controls when and where in an animal a gene is expressed.

Pronucleus: structure containing genetic materials from the egg or sperm in a recently fertilized egg.

Quantitative traits: genetic traits controlled by many genes that influence production such as growth rate, fertility and milk production.

Site-directed mutagenesis: an introduced change in a gene at a specific, targeted site in the gene.

Transgene: genetic material introduced by means other than natural breeding (an animal carrying a transgene is referred to as being transgenic). passed to a transgenic animal's progeny requires that the original transgenic animal reach sexual maturity and then complete at least one gestation period. This could take several years in some species.

Microinjection of DNA into the pronuclei of recently fertilized ova is the most common technique used to produce genetically engineered livestock, but on average fewer than $2 \%$ of microinjected embryos yield transgenic individuals. Furthermore, pronuclear microinjection has allowed only the random integration of a transgene into the animal genome.

Since 1980 , scientists have made continual advances in the efficiency of producing embryos from farm animals in the laboratory, called in vitro maturation and fertilization. These techniques, combined with improved conditions for embryo culture and cryopreservation and increased experience and sophistication in the production and manipulation of embryos, culminated in the cloning of an adult sheep, "Dolly," by Scottish scientists in 1997 (Wilmut et al. 1997).

Advances in animal cloning procedures, beginning with transgenic donor cells, have also resulted in the production of transgenic sheep, goats and cattle. Although today's overall efficiency appears to be comparable to that of pronuclear microinjection, cloning to produce transgenic livestock will allow the insertion of DNA sequences - as embryonic stem cells do in mice - creating the opportunity to alter or remove a specific gene (fig. 1).

With continued scientific improvements, the production of transgenic livestock through cloning could eventually be more efficient than pronuclear microinjection. Modern biotechnology can be used to improve the genetic merit of livestock by two routes: transferring novel genetic material via genetic engineering or accelerating the dissemination of desirable traits via the cloning of selected individual animals.

\section{Cloning and genetic improvement}

The successful cloning of an adult sheep (Wilmut et al. 1997) captured 
the imagination of both the scientific community and the rest of the world. Dolly - the sheep cloned from a cultured mammary gland cell of a 6-yearold ewe long since dead - made the cover of most major news and scientific magazines and became the subject of global commentary and debate. Lost in the debate was a primary reason behind the investigators' experiments: the identification of cell types that could be genetically manipulated in the laboratory and subsequently used to generate a transgenic animal.

Developments in cloning have taken two forms since Dolly's birth: demonstrations of the practical uses of cloning to produce transgenic animals, and the expansion of the species and types of cells that can be used as nuclear donors in successful cloning. Transgenic fetal fibroblasts - isolated and engineered from connective tissue cells - have been used to clone transgenic lambs, calves and goat kids.

Successful cloning from adult cells has been expanded to include various cell types in cattle and laboratory mice. The potential to clone adult animals creates entirely new dimensions for animal agriculture. A desirable and unique specimen can be precisely reproduced, capturing traits that are difficult to develop through traditional breeding practices. For example, a dairy cow that produces milk with unusually high milk protein content (which is important for making cheese), or with an unusually low percentage of saturated fat (which has human health benefits), could be cloned. Selective breeding from an individual animal is not always successful, but selective breeding from a nucleus core herd of cloned animals is more likely to succeed.

An example of the power of cloning is the preservation of the last surviving cow of the Australia's Enderby Island cattle breed. Cloning of this female and use of available cryopreserved semen to breed the surviving clones could provide a second chance to resurrect a breed that otherwise would have disappeared.

We can only speculate about what impact cloning technology will have

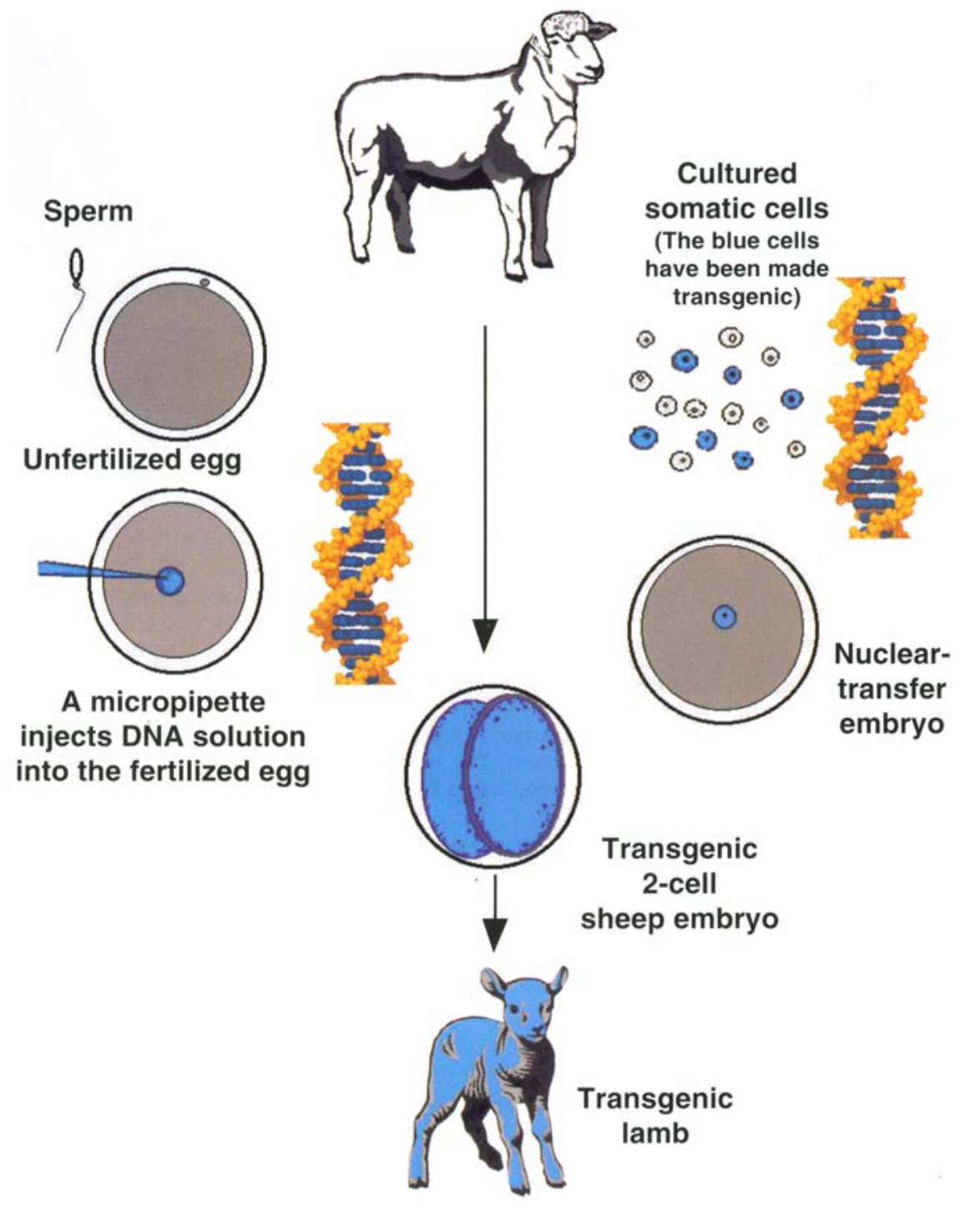

Fig. 1. Transgenic farm animals are produced by direct microinjection of DNA into young embryos and by cloning from transgenic somatic cells. Microinjection procedures use recently fertilized eggs, which for some species can be obtained from in vitro fertilization procedures, before the first cell division. If the foreign DNA becomes integrated into the embryonic genome at the one-cell stage, as the embryo develops all of its cells will contain the transgene. The offspring that is born after transfer of the embryo to the reproductive tract of a recipient female will be transgenic. Alternatively, somatic cells can be collected from an animal, cultured in the laboratory, and exposed to foreign DNA. Some cells will become transgenic, and these cells can be selected for use as nuclear donors in nuclear-transfer procedures. The resulting nuclear-transfer embryo will be transgenic, as will the offspring born after embryo transfer and term development in the reproductive tract of a recipient female.

on genetic improvement, but potential impacts could be considerable for intensively managed systems such as the dairy industry. Early in the 21st century, cloning could be used to duplicate the top-producing dairy cows. Artificial insemination eliminates the need to clone elite bulls, but at present each elite cow can produce only a limited number of offspring, even with the use of embryo transfer technology.

The ability to clone genetically elite females, while possibly increasing the level of inbreeding, also increases the intensity of genetic selection. By 2025, cloning and breeding of these cloned animals could be carried out by companies comparable to the current artificialinsemination industry, which is responsible for selection and breeding 


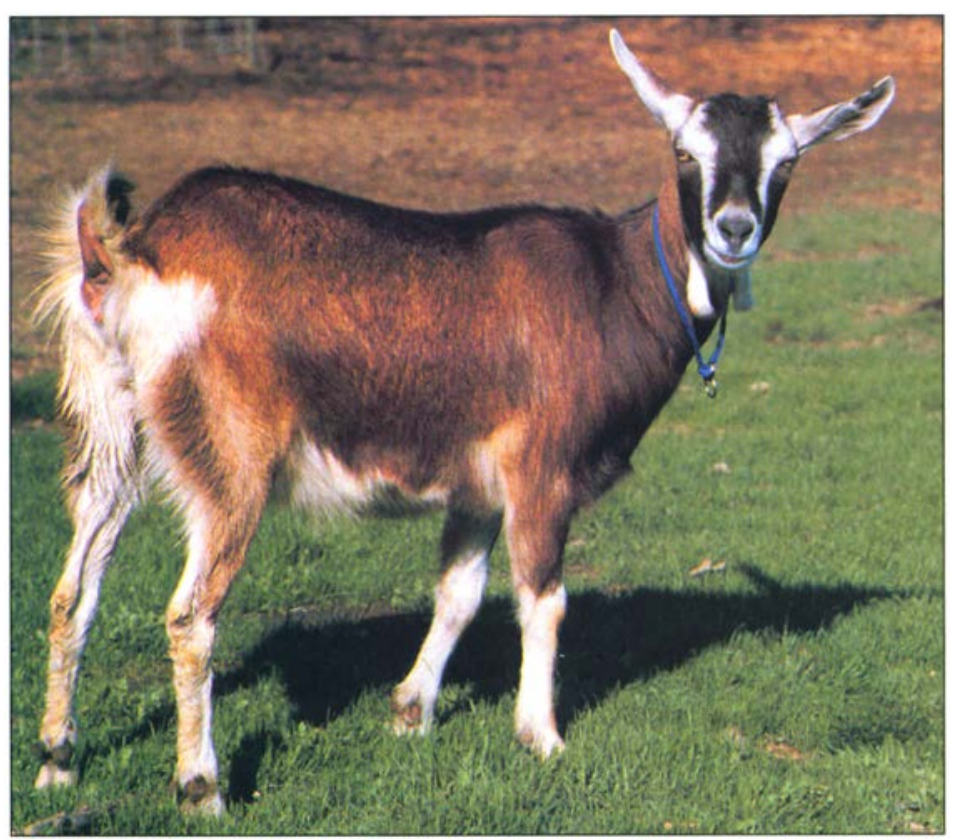

This transgenic goat has a transgene that codes for a human protein under the control of a promoter region that targets expression specifically to the mammary gland. The human protein is secreted in the goat's milk but nowhere else in the animal.

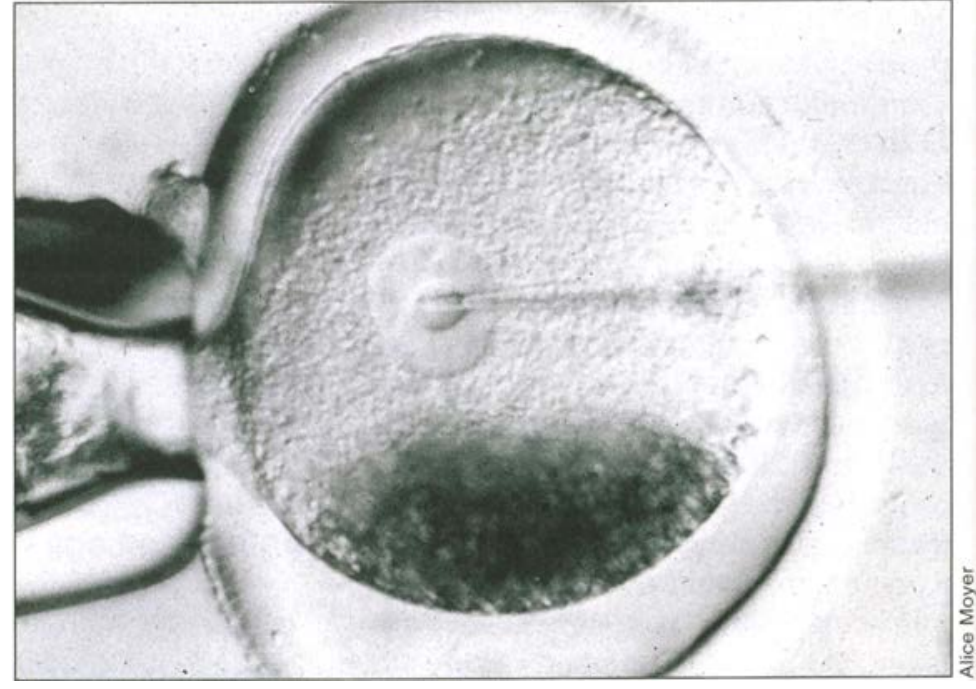

A recently fertilized bovine embryo (zygote) is microinjected to introduce a DNA solution into its genetic material. Microinjection of zygotes is currently the most common method to produce transgenic animals. of the top dairy stock. The swine industry could undertake a similar strategy for the expansion of the cloned lines of top breeding sows.

\section{Livestock with improved traits}

During the 15 years since the first transgenic farm animals were produced, the rationale for genetic engineering of livestock for agricultural purposes has been to produce animals with altered traits such as disease resistance, wool growth, body growth or milk composition. In most instances, the objective has been either to alter traits for improved production efficiency or to alter the properties of the animal product, such as wool or milk, and increase the range of manufacturing options.

Gene constructs - designed to express directly or indirectly various growth factors and alter body composition - constitute the largest class of transgenes transferred into livestock species. The majority of these transgenes expressed growth hormone $(\mathrm{GH})$, although other constructs based on GH release and insulin-like growth factor-I (IGF-I) also have been used. In general, pigs and sheep expressing these constructs were leaner and more feed-efficient. But as a result of high, unregulated levels of circulating GH, they also suffered a number of complications, such as joint problems, indicating the need for tight control of hormone secretion.

Recently, two research groups reported preliminary data on the development of GH and IGF-I transgenic pigs with enhanced growth-performance traits (Nottle et al. 1999; Pursel et al. 1999). In both experiments, desirable effects on growth and body composition traits were achieved without apparent abnormalities, suggesting that someday useful animals could become available to swine breeders. Potentially useful GH-transgenic fish also have been produced, but biological containment of the transgene is of great concern in species with existing wild fish populations.

Milk protein genes have been cloned from a variety of mammals. The promoter elements from certain milk-protein genes from one or more species have been used to facilitate expression of transgenes in the mammary glands of mice, sheep, goats, cattle, rabbits and pigs (Murray and Maga 1999). These transgenes are developmentally correct, but their levels of expression can vary. Research on targeting transgene expression to the mammary gland of farm animals either has focused on studying the promoter function or on the production and recovery of biologically important, active proteins for use as pharmaceuticals (Maga and Murray 1995).

Several private companies have produced transgenic cows, sheep, goats and pigs, targeting transgenic expression to the mammary gland with the aim of isolating high-value pharmaceutical proteins from milk. But the use of transgenesis for agricultural purposes, such as to alter the properties and composition of milk and change the functional properties of the milk protein system, is also possible by adding a new gene or altering an existing gene.

We have produced transgenic mice that express human lysozyme or a modified bovine casein (a protein used in cheese-making and for some industrial purposes) in the milk. As a result, we have measured alterations in the physical and functional properties of the mouse's milk protein system, including decreased micelle size and increased gel strength. The production of human lysozyme in milk of transgenic mice also increased the antimicrobial properties of the milk, which in cows could reduce infections 


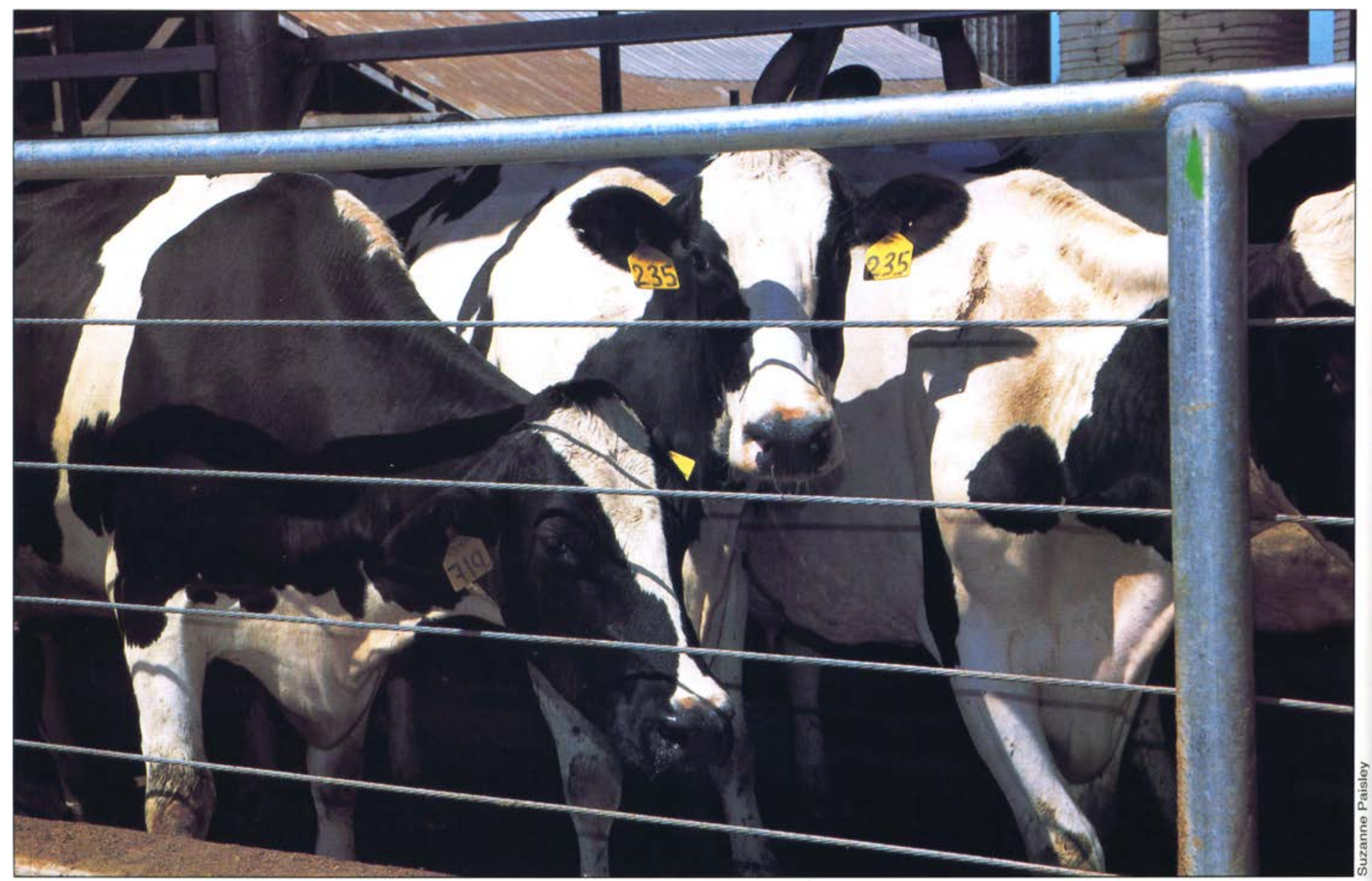

Within 20 years, transgenic and cloned animals could become useful in production agriculture. For these technologies to be commercialized, issues such as the integration of transgenes into breeding populations, genetic diversity and inbreeding, and industry and consumer acceptance must be addressed.

in the mammary gland and perhaps eliminate undesirable pathogens in the gut of humans who consume the milk.

\section{Alternative scenarios}

Two scenarios demonstrate how genetic engineering can be applied to improve livestock. The first involves altering milk to improve functionality and human health. In the second scenario, genetic engineering is used to reduce environmental pollution stemming from animal agriculture and aquaculture. Other useful applications are possible; perhaps diseases such as scrapie and "mad cow disease" could be eliminated by deleting the gene leading to the disease.

Milk improvements. Variations in the composition and functionality associated with milk proteins, such as leading to more efficient cheese-making or new types of cheese, suggest that. changes in these properties should be possible. Richardson and colleagues proposed specific alterations in the properties of milk that might be achieved by overexpressing, deleting or adding back a mutated form of most major milk protein genes (Jimenez-Flores and Richardson 1988; Yom and Richardson 1993). For example, adding extra copies of the casein gene to overexpress casein could increase the thermal stability of milk, reducing protein breakdown during manufacturing.

A more complex proposal would be to alter a casein gene at a specific site (called site-directed mutagenesis) prior to gene transfer. The presence of $10 \%$ to $20 \%$ of the altered casein in milk produced by a transgenic cow could increase proteolysis (e.g., protein breakdown) and thereby promote the faster ripening of cheese. Results of experiments with transgenic mice illustrate the positive effects of adding genes such as casein (Gutiérrez-Adán et al. 1996) or human lysozyme (Maga et al. 1997) to the milk protein system.

Ongoing research, supported in part by the industry's California Dairy Research Foundation, is exploring potential uses of transgenic technology in the dairy industry. We expect genetically engineered dairy cows to become available within two decades, includ-

We expect genetically engineered dairy cows to become available within two decades, including animals that produce greater cheese yields and healthier milk for human consumption, as well as a wider range of milk products. 
ing animals that produce greater cheese yields and healthier milk for human consumption, as well as a wider range of milk products.

For example, the increased expression of certain milk proteins could yield animals that produce milk specifically for manufacturing and industrial purposes, such as for cheese-making versus fluid-milk consumption. Alternatively, our research program is designed to improve the nutritional and antimicrobial properties of milk intended for human consumption. Our laboratory at UC Davis has already shown that human lysozyme, when expressed as a transgene in mice, maintains antimicrobial activity, some of which is enhanced when lysozyme is secreted by the mammary gland versus simply adding lysozyme to milk (Maga et al. 1997).

Experiments are currently underway to add other naturally occurring human milk proteins - also having antimicrobial properties - and genes to alter the fatty-acid composition of milk in favor of a more heart-healthy mix.

Dairy cows carrying these types of transgenes could become available by 2025. Using transgenic cows could result in the gradual separation of the genetic backgrounds of herds being used for fluid milk production from those used for producing milk for cheese manufacturing. For example, the antimicrobial properties of lysozyme-containing milk for drinking could interfere with the microbes used in cheese and yogurt production.

Greater specialization among dairy herds could result, with some herds earning premiums for producing specific types of milk for particular niche markets.

Environmental protection. Livestock production, particularly intensive systems like dairy, swine, poultry and aquaculture, needs to reduce the amount of minerals excreted by animals. Because the digestive processes of livestock can be inefficient, compounds such as phosphate are usually added to feed at levels exceeding the animals' dietary requirements. These minerals accumulate at elevated levels in surface water and groundwater, damaging aquatic life and contaminating drinking water.

Reducing phosphate pollution of water is a major challenge for swine, poultry and finfish producers. To the extent that enzymes can be added to increase the efficiency of feed additives, the amount of that additive in the feed can be reduced. If a more effective phytase (an enzyme that breaks down phytic acid, a phosphatecontaining ring compound produced by plants) can be expressed in the animal's digestive tract, then the amount of phosphate in the diet can be re-

duced, and this in turn would lower the amount of unused phosphate it excretes. Such "environmentally friendly," genetically modified animals could become available to various livestock industries within 10 to 15 years.

\section{Issues and concerns}

The potential application of cloning and genetic engineering technology to livestock raises a number of important issues.

Integration of a transgene into a breeding population. Transgenic animals could become useful in production agriculture within 20 years. However, production of a useful transgenic line is only the first step toward introducing the transgene into a production population.

To begin with, the transgene must be transferred to core breeding herds, and then the herds must undergo selection to optimize performance for the desired traits. This will be particularly important for transgenes affecting quantitative traits such as growth, body composition and reproduction, and for transgenes that modify intermediary metabolism.

Research on the integration of a transgene into a breeding population using a model system has begun only recently. The introgression of a transgene into livestock will be expensive, due to the cost of breeding. A second cost stems from the lack of selection and thus genetic improvement during the period when the transgene is being inserted and integrated into the breeding population. 


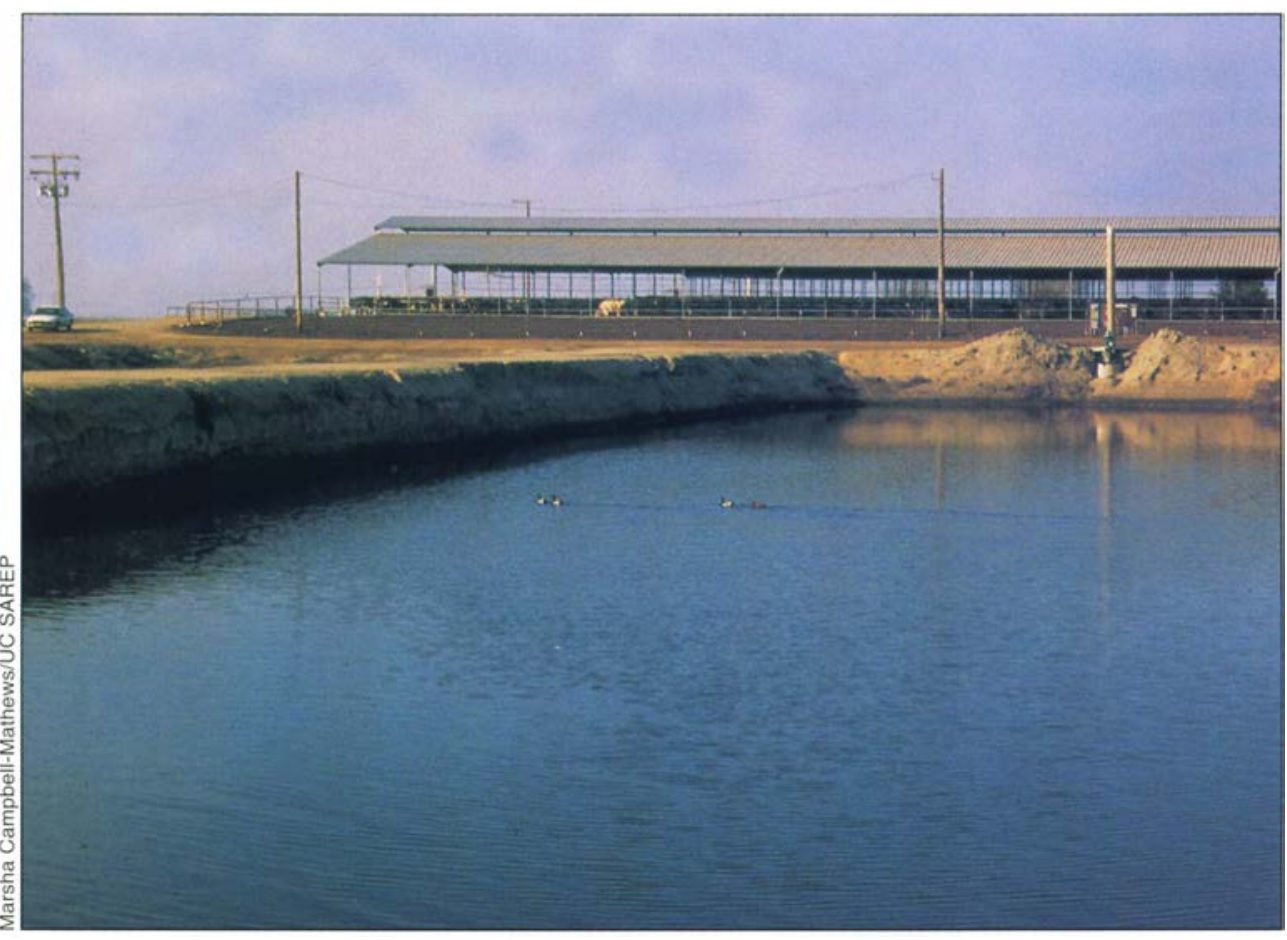

Genetic engineering may be able to reduce groundwater pollution by improving enzymes in the digestive tracts of Ilvestock animals, thereby reducing the amount of phosphates they must consume and excrete. Lagoons are one of the practices currently employed by dairies to manage animal waste.

Gama et al. (1992) suggested that the best strategy for introgressing a transgene into a core swine herd would involve three generations of backcrossing before selecting a herd and evaluating their characteristics. Backcrossing involves crossing an offspring with one of two parental populations. The economic benefits of the transgene affecting a production trait or production efficiency must be sufficiently high to compensate for these costs.

Recent results of research with GHtransgenic mice show that the heritability of various body-composition traits is altered and that the traits that result from transgene expression may be dependent on the genetic background of the strains or breed line (Siewerdt et al. 1999). It cannot be assumed that the transgene will necessarily yield the same desired traits when placed in different genetic backgrounds.

Genetic diversity and inbreeding. Another potential concern is whether the use of cloning or genetically engineered animals will lead to reductions in genetic diversity or increased rates of inbreeding among livestock. $A$ priori there is no reason to assume that either of these impacts will result to a greater extent than when conventional breeding techniques are used.

If cloned animals, which by definition exhibit virtually no genetic variation, are properly used within the context of a selective breeding program, then inbreeding should be minimized. The same is true for transgenic animals, which are more inbred than the population at large, whether produced by microinjection or cloning. In either case, the reliance on a limited number of founder animals could lead to increased inbreeding, while the selection by industry of a limited number of production genotypes, like breeds today, could lead to reduced genetic diversity in the gene pool over a prolonged period. Maintenance of genetic diversity is desirable to allow future improvements through breeding for production and health traits.

Industry acceptance. The acceptance of genetically engineered animals by industry, as with the use of cloning to reproduce exceptional fe- males, will depend on economic incentives. If the cost of stock or loss in selection progress is greater than the return to the producer through increased efficiencies or income over a reasonable period, producers will not use these technologies.

In cases where the transgene results in new products, such as antimicrobial milk or moth-resistant wool, the producer would probably need to obtain a premium price to convert the production flock or herd to the new genotype. As with antimicrobial milk, the introduction of some new genotypes may lead to segmenting the industry and creating special uses for different populations of animals so that new "breeds" are established. In the end, if scientists have done a good job in selecting traits to be manipulated, the acceptance of genetically modified animals by industry will come down to whether or not consumers are prepared to buy the resulting products.

Consumer acceptance. Debate continues in Europe, Japan and the United States over the use of genetically modified crops. In the United States, a number of genetically modified crops have been approved by the U.S. Department of Agriculture for commercial use. There is every reason to assume that the introduction of genetically modified livestock will engender similar public debate. Concerns range from decreasing genetic diversity and the safety of genetically modified foods to animal welfare issues.

For genetically modified crops in cultivation, plants have been modified to resist herbicides or insect pests. The traits that have been genetically engineered into plants so far are similar to the growth-enhancing transgenes in animals. Because they affect production traits rather than the quality of the final product, consumers may not perceive any direct benefits to them or may believe the benefits are not worth the perceived costs.

\section{Future in focus: Adoption depends on cost}

Biotechnology has contributed to the genetic improvement of farm animals for decades, through artificial in- 
semination and embryo transfers. Transgenic technology and cloning can, and indeed should, be successfully used to increase the genetic merit of livestock. Transgenic sheep, goats, pigs and cattle can now be made routinely, although the efficiency still is low and the costs high, particularly for cattle.

Sheep, goats, pigs and cattle have been successfully cloned, but again the efficiency is low. While the cost of production is not a major concern when producing transgenic animals for biomedical or pharmaceutical production purposes, it poses a considerable barrier to the introduction of these techniques for production animals in agriculture, particularly given the low level of public funding for animal agricultural research (see p. 72).

The advent of cloning as a method for producing genetically engineered animals may increase our ability to produce transgenic livestock. Because the transgene is inserted during the cell-culture phase, each offspring born will be transgenic, and each will have the same insertion site. If the efficiency of cloning is improved, then the efficiency of producing transgenic animals will also be increased. The fact that transgenic animals are clones is relevant only to the extent that care must be taken to avoid inbreeding. The ability to re-derive animals from cells in culture finally opens up the possibility of doing experiments to eliminate undesirable genes and traits in livestock (called gene knockout or gene replacement).

The results of recent work with growth-enhanced transgenic pigs indicate that it is possible to control systemic-acting transgenes so that desirable effects are obtained without the health impairments seen in earlier experiments. The mammary gland and the milk protein systems are robust and can be altered and added to using a variety of different proteins and will still function normally. Furthermore, these systems can be altered to produce predictable changes in the functional and antimicrobial nature of milk.

Current research suggests that animals potentially useful for commercial production may already be available, while results from transgenic mice demonstrate the potential to manipulate milk and improve its properties for manufacturing and human consumption (Murray et al. 1999). Genes and promoters are being identified on a massive scale through various genome mapping and functional genomics initiatives.

While commodities such as milk and fiber can be genetically modified, many economically important production traits, such as growth, require genetic modification of basic metabolic systems. Until recently, research on the most appropriate strategies and potential problems arising from the introgression of transgenes into production populations with different genetic backgrounds has largely been ignored.

We are optimistic about the future of transgenic animals in agriculture. We predict that by midcentury most agricultural animals will be genetically engineered to be more efficient and healthier than current stock, producing healthy products for human consumption in an environmentally friendly system.

Although the low efficiency of current genetic engineering technology is a limiting factor, the need to carry out selection to optimize the performance of transgenic production animals may be an even greater limiting factor in the development of commercial herds.

The cost of producing suitable transgenic animals for use in production herds versus the potential economic benefits could limit the use of such animals. As we move into the 21st century, we must engage in two dialogues: one with the agricultural animal industry to determine the most important areas to target for manipulation and another with the public so that consumers fully understand the nature of the genetic changes being introduced.

\footnotetext{
J.D. Murray is Professor, Department of Animal Science, College of Agricultural and Environmental Sciences, and Department of Population Health and Reproduction, School of Veterinary Medicine, UC Davis. G.B. Anderson is Professor and Chair, Department of Animal Science, UC Davis.
}

\section{References}

Gama LT, Smith C, Gibson JP. 1992. Transgene effects, introgression strategies and testing schemes in pigs. Anim Prod 54:427-40.

Gordon JW, Scangos GA, Plotkin DJ, et al. 1980. Genetic transformation of mouse embryos by microinjection of purified DNA. Proceedings of the National Academy of Sciences USA 77:7380-4.

Gutiérrez-Adán A, Maga EA, Meade HM, et al. 1996. Alteration of physical characteristics of milk from bovine kappa-casein transgenic mice. J Dairy Sci 79:791-9.

Jimenez-Flores R, Richardson T. 1988.

Genetic engineering of the caseins to modify the behavior of milk during processing: $A$ review. J Dairy Sci 71:2640-54.

Maga EA, Murray JD. 1995. Mammary gland expression of transgenes and the potential for altering the properties of milk. Bio/ Technology 13:1452-7.

Maga EA, Anderson GB, Cullor JS, et al. 1997. Antimicrobial properties of human lysozyme transgenic mouse milk. J Food Protection 61:52-6.

Murray JD, Maga EA. 1999. Changing the composition and properties of milk. In: Murray JD, Anderson GB, Oberbauer AM, McGloughlin MM (eds.). Transgenic Animals in Agriculture. Wallingham, UK: CAB International. 304 p.

Murray JD, Anderson GB, Oberbauer AM, McGloughlin MM (eds.). 1999. Transgenic

Animals in Agriculture. Wallingham, UK: CAB International. $304 \mathrm{p}$.

Nottle MB, Nagashima $H$, Verma PJ, et al. 1999. Production and analysis of transgenic pigs containing a metallothionein porcine growth hormone gene construct. In: Murray JD, Anderson GB, Oberbauer AM,

McGloughlin MM (eds.). Transgenic Animals in Agriculture. Wallingham, UK: CAB International. $304 \mathrm{p}$.

Pinkert CA, Murray JD. 1999. Transgenic farm animals. In: Murray JD, Anderson GB, Oberbauer AM, McGloughlin MM (eds.).

Transgenic Animals in Agriculture.

Wallingham, UK: CAB International. $304 \mathrm{p}$.

Pursel VG, Wall RJ, Mitchell AD, et al. 1999. Expression of insulin-like growth factorI in skeletal muscle of transgenic swine. In: Murray JD, Anderson GB, Oberbauer AM, McGloughlin MM (eds.). Transgenic Animals in Agriculture. Wallingham, UK: CAB International. 304 p.

Siewerdt F, Eisen EJ, Murray JD. 1999. Direct and correlated responses to short-term selection for 8-week body weight in lines of transgenic (oMt1a-oGH) mice. In: Murray JD, Anderson GB, Oberbauer AM, McGloughlin MM (eds.). Transgenic Animals in Agriculture. Wallingham, UK: CAB International. $304 \mathrm{p}$.

Wall RJ, Hawk HW, Nel N. 1992. Making transgenic livestock: Genetic engineering on a large scale. J Cell Biochem 49:113-20.

Wilmut I, Schnieke AE, McWhir J, et al. 1997. Viable offspring derived from fetal and adult mammalian cells. Nature 385:810-3.

Yom HC, Richardson T. 1993. Genetic engineering of milk composition: modification of milk components in lactating transgenic animals. Amer J Clin Nutr 58:299S-306S. 